\title{
gs
}

\section{Single-Photon-Level Quantum Memory at Room Temperature}

\author{
K. F. Reim, ${ }^{1, *}$ P. Michelberger, ${ }^{1}$ K. C. Lee, ${ }^{1}$ J. Nunn, ${ }^{1}$ N. K. Langford, ${ }^{1}$ and I. A. Walmsley ${ }^{1}$ \\ ${ }^{1}$ Clarendon Laboratory, University of Oxford, Parks Road, Oxford OX1 3PU, United Kingdom
}

(Received 23 November 2010; published 25 July 2011)

\begin{abstract}
Room-temperature, easy-to-operate quantum memories are essential building blocks for future long distance quantum information networks operating on an intercontinental scale, because devices like quantum repeaters, based on quantum memories, will have to be deployed in potentially remote, inaccessible locations. Here we demonstrate controllable, broadband and efficient storage and retrieval of weak coherent light pulses at the single-photon level in warm atomic cesium vapor using the robust far off-resonant Raman memory scheme. We show that the unconditional noise floor of this technically simple quantum memory is low enough to operate in the quantum regime, even in a room-temperature environment.
\end{abstract}

DOI: 10.1103/PhysRevLett.107.053603

PACS numbers: 42.50.Ct, 03.67.Hk, 42.50.Ex

In an age of ever-increasing, global information transfer, there is growing demand for secure communication technology, such as could be provided by photonic quantum communications networks [1]. Currently, the biggest challenge for such networks is distance. Over short distances, photons, interacting only weakly with their environment, easily and reliably carry quantum information without much decoherence, but intercontinental quantum communication will require quantum repeaters embedded in potentially isolated locations, because photon loss rises otherwise exponentially with distance [2,3]. In general, these repeaters will require some sort of quantum memory, a coherent device where single photons are reversibly coupled into and out of an atomic system, to be stored, possibly processed and then redistributed. In order to be practically useful, this will need to have sufficiently large bandwidth, high efficiency and long storage time, with multimode capacity, and a low-enough noise level to enable operation at the quantum level. Furthermore, to be truly practical, a critical feature of such memories will be ease of operation near room temperature for use in isolated, potentially unmanned locations.

Warm atomic vapors of alkali atoms such as rubidium and cesium are potentially excellent storage media. At moderate temperatures the vapor pressure is sufficient to render the doublet lines optically thick, providing a strong atom-photon interaction in a centimeter-scale vapor cell. With the addition of a buffer gas to slow atomic diffusion, collective atomic coherences on the order of milliseconds have been observed [4]. There is no need for laser trapping, high vacuum or cryogenic cooling, and atomic vapor cells can be miniaturized [5], integrated onto chips using hollow-core waveguide structures [6] and mass-produced. Cost-effective integration is likely to be crucial for quantum photonics to develop into a mature technology.

Previously, nonclassical continuous variable states have been mapped into warm atomic vapor via a weak interaction using measurement and feedback [7]. Applications such as quantum repeaters [2], however, require reversible quantum memories-i.e., where the excitation can be reversibly retrieved on demand. This requires strong lightmatter coupling, which can then introduce noise. An example of where this type of strongly coupled interaction can be achieved is electromagnetically induced transparency (EIT), which has been used to demonstrate storage and reversible retrieval of classical signals [8], heralded single photons [9], and squeezed vacuum [10] in warm atoms. In these experiments the noise was either not resolved, not measured or estimated only in the context of highly selective postprocessing. By contrast, studies which have focussed on noise in EIT have concluded that collision-induced fluorescence gives rise to an unconditional noise floor which is too high to be compatible with quantum applications [11,12]. Here, however, we demonstrate a memory protocol that operates with very low noise.

Using a fundamentally different approach, i.e., the recently developed far off-resonant Raman scheme [13], we demonstrate the first room-temperature "stopped-light" memory that operates with a low unconditional noise floor in the quantum regime. We also show that the memory fulfils all requirements for true quantum operation in a robust and simple room-temperature architecture. Specifically, we demonstrate total memory efficiencies of $\eta>30 \%$, and with only moderate magnetic shielding, we show storage times of up to a maximum of $4 \mu \mathrm{s}$, around 2500 times longer than the duration of the pulses themselves. This is the largest time-bandwidth product demonstrated to date and is already sufficient to improve heralded multiphoton rates from parametric down-conversion sources. Finally, we make a detailed analysis of the unconditional noise floor of the memory, which is found to be $<0.25$ photons per pulse, that is, low enough for quantum applications. The combination of these results shows that we have a quantum-ready memory, capable of handling quantum information in a simple room-temperature design. 
Issues of noise are largely avoided in cold-atom experiments [14-19], where atomic collisions are suppressed or eliminated. But the technical complexity of these experiments, which require the trapping and cooling of atomic clouds under high vacuum, makes large-scale deployment of this type of memory rather challenging, especially outside controlled laboratory conditions. Similarly, solid-state memories are promising candidates for efficient and lownoise photon storage [20,21], but currently they must be operated at cryogenic temperatures.

By contrast, ensemble-type Raman memories which are tuned far from resonance [22] provide a possible path to fulfilling all of the requirements for quantum-ready operation at room temperature. The far off-resonant Raman interaction results in (i) extremely broadband capability, allowing us to interface the memory with conventional parametric down-conversion sources [23], (ii) the ability to optically switch the memory in and out of the quantum channel, or alternatively set the storage level to $50 \%$, providing a straightforward noninterferometric path to creating light-atom entanglement, (iii) very weak fluorescence noise which is predominantly nonsynchronous with the short signal pulse, and (iv) memory efficiency which is insensitive to inhomogeneous broadening, allowing roomtemperature operation and a path towards integrated implementations. Apart from the advantage of simplicity [24], operating at room temperature also makes it easier to achieve larger optical depths, and hence higher efficiencies $[22,25]$.

The heart of our Raman quantum memory $[13,22]$ is a cesium-vapor atomic ensemble [26], prepared in a vapor cell heated to $62.5^{\circ} \mathrm{C}$, that makes use of the $852-\mathrm{nm} D_{2}$ line, with the $6^{2} S_{1 / 2}$ hyperfine states serving as the ground $|1\rangle$ and storage $|3\rangle$ states [Fig. 1(a)]. The atomic ensemble is initially prepared in the ground state $|1\rangle$ by optical pumping with an external cavity diode laser (ECDL). At $t_{1}$, the signal gets mapped by the strong write field into a collective atomic spin-wave excitation, and at $t_{2}$, a strong read pulse reconverts the atomic coherence back into the photonic mode [Fig. 1(b)]. For the storage process, the signal and write pulses, both derived from a Ti:Sapph laser oscillator and an electro-optic modulator [13], are sent temporally and spatially overlapped into the vapor cell [Fig. 1(b)]. The pulses have a duration of $300 \mathrm{ps}$ corresponding to a bandwidth of $1.5 \mathrm{GHz}$. A Pockels cell is used for selecting the desired pulses from the $80 \mathrm{MHz}$ pulse train of the laser oscillator. The bandwidth of the memory is defined dynamically by the strong write field which is about $10^{10}$ times brighter than the single-photon-level signal field. The weak signal is then separated from the strong write and read fields via polarization and spectral filtering, amounting to a total extinction ratio of $10^{-11}$ [26]. This filtering is particularly important when operating at the single-photon level and is provided by a Glan-laser polarizer and three air-spaced Fabry-Perot etalons with a free spectral range of $18.4 \mathrm{GHz}$ and transmission window of $1.5 \mathrm{GHz}$. Note that the free

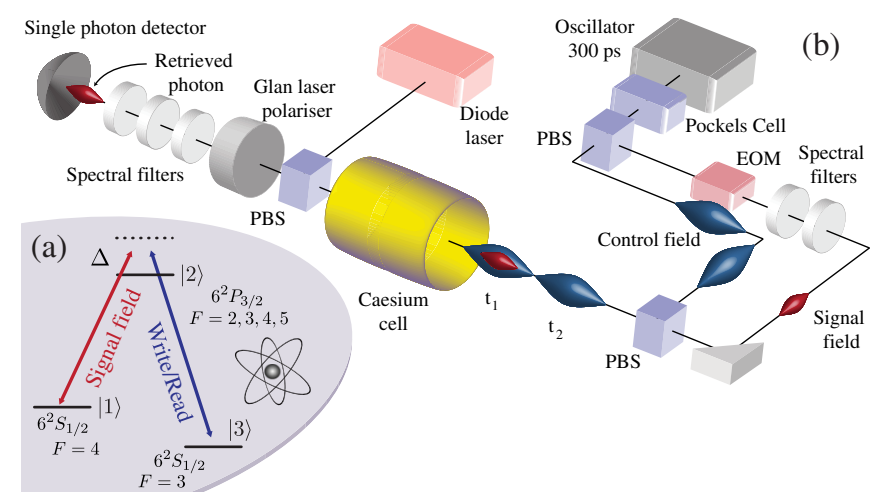

FIG. 1 (color online). (a) $\Lambda$-level scheme for the Raman memory. $\Delta$ is the detuning from the atomic resonance. (b) Experimental setup. At $t_{1}$ the single-photon-level signal is mapped by a strong write pulse into a spin-wave excitation in the cesium vapor cell. At $t_{2}$ a strong read pulse reconverts the excitation into a photonic mode. After polarization filtering with a PBS (polarizing beam splitter) and a Glan-laser polarizer as well as spectral filtering with Fabry-Perot etalons, the retrieved signal is detected with a single-photon detector (SPCM). The control field is vertically polarized and the signal field is horizontally polarized.

spectral range is twice the $9.2 \mathrm{GHz}$ Stokes shift, so both Stokes (signal) and anti-Stokes (noise) frequencies pass through the filters. As discussed in more detail below, anti-Stokes noise could be eliminated using more selective filters. Finally, the signal field is detected using a silicon avalanche photodiode. In these experiments, the memory is operated at a repetition rate of $3 \mathrm{kHz}$.

In the current experiment, we demonstrate total efficiencies exceeding $30 \%$ and a memory lifetime $(1 / e$ characteristic time) of around $1.5 \mu \mathrm{s}$ (Fig. 2), which is double the efficiency and more than 2 orders of magnitude improvement in lifetime over the values obtained in our previous experiment [13]. These results were obtained with only moderate magnetic shielding and residual static fields are the main dephasing mechanism [26]. In principle, magnetic dephasing can be eliminated by improved shielding, enabling storage times limited by atomic diffusion to several hundred $\mu \mathrm{s}$ [27]. However, because this memory has such a broad bandwidth, this measured lifetime already corresponds to a time-bandwidth product of $\sim 2500$. This is the largest time-bandwidth product of any quantum memory demonstrated so far, and is already sufficient to dramatically improve multiphoton rates generated by heralded parametric sources. As discussed in Ref. [13], the memory efficiency is restricted mainly by control field power and the less efficient, but experimentally simpler, forwardretrieval configuration [22].

Next, we measure the memory output using a singlephoton counting module (SPCM; a Geiger mode silicon avalanche photodiode) to test its performance at the single-photon level. The SPCM is combined with a time-to-amplitude converter and a multichannel analyzer, which allows photodetection events to be time-binned with a resolution of $\sim 125$ ps. Figure 3(a) shows storage at 


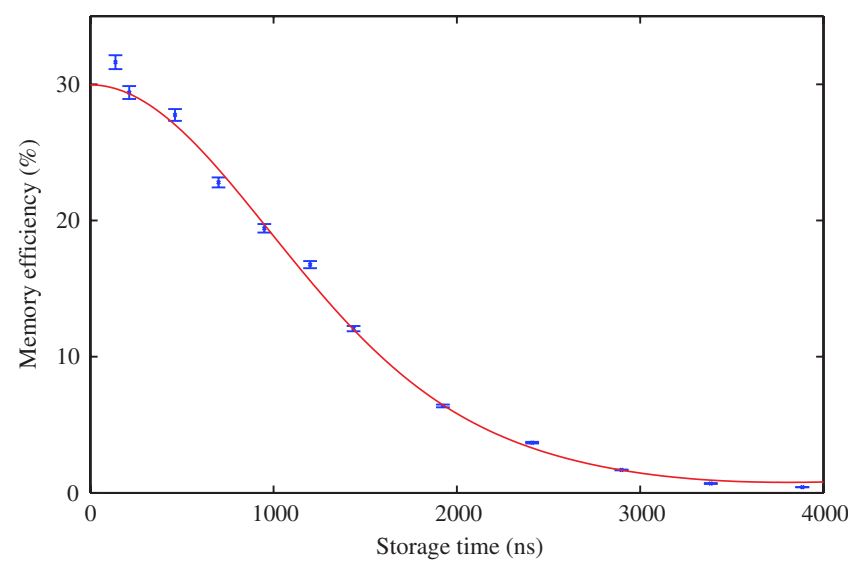

FIG. 2 (color online). Memory efficiency. The lifetime of the memory is about $1.5 \mu \mathrm{s}$. The blue dots indicate experimental data measured with a fast APD. Error bars represent the standard error in the mean. The solid line is the theoretical dephasing predicted for a constant magnetic field of $0.13 \pm 0.05$ Gauss, which can be attributed to the residual of the Earth's magnetic field [26]. Note that this dephasing could be compensated using spin-echo techniques, or suppressed with improved magnetic shielding.

$t=0 \mathrm{~ns}$ and retrieval at $t=780 \mathrm{~ns}$ later for an average input signal of 1.6 photons per pulse $(<1$ retrieved photon per pulse), while Figs. 3(b) and 3(c) show the storage and retrieval processes in detail: the plots show histograms derived from 360000 experiments. The strong reduction in transmitted signal [Fig. 3(b)] between the control field being off (green [light gray]) and on (blue [dark gray]), and the significant amount of retrieved signal [Fig. 3(c), blue (dark gray)] demonstrates that the quantum memory operates well at the single-photon level, even when retrieving at a delay of $>1500$ times the signal pulse duration. However, to properly characterize the noise in these signals, it is critical to measure the unconditional noise floor: the signal detected when memory retrieval is triggered without any stored input signal (Figs. 3(b) and 3(c), red [medium gray] curves). In our case, this is the detected signal when the control field is sent in with no input signal, and is measured to be 0.25 photons per pulse. To assess the effects of this noise in a quantum application, we consider a simple thought experiment based on the Duan-Lukin-CiracZoller quantum repeater protocol. By storing one half of an entangled state and averaging correlations over signal and noise photons retrieved from the memory, one can show that this noise level is already low enough to maintain the security of quantum key distribution via the Ekert protocol [28], in which violation of Bell's inequality certifies the quantum channel [26]. The measured noise floor is also low enough to allow the storage and retrieval of polarization qubits with fidelity exceeding that of the best possible classical memory [26,29].

We now explore the origin of the noise photons to determine if the observed noise could be further reduced. The inset to Fig. 3(a) shows that the retrieved signal peak is
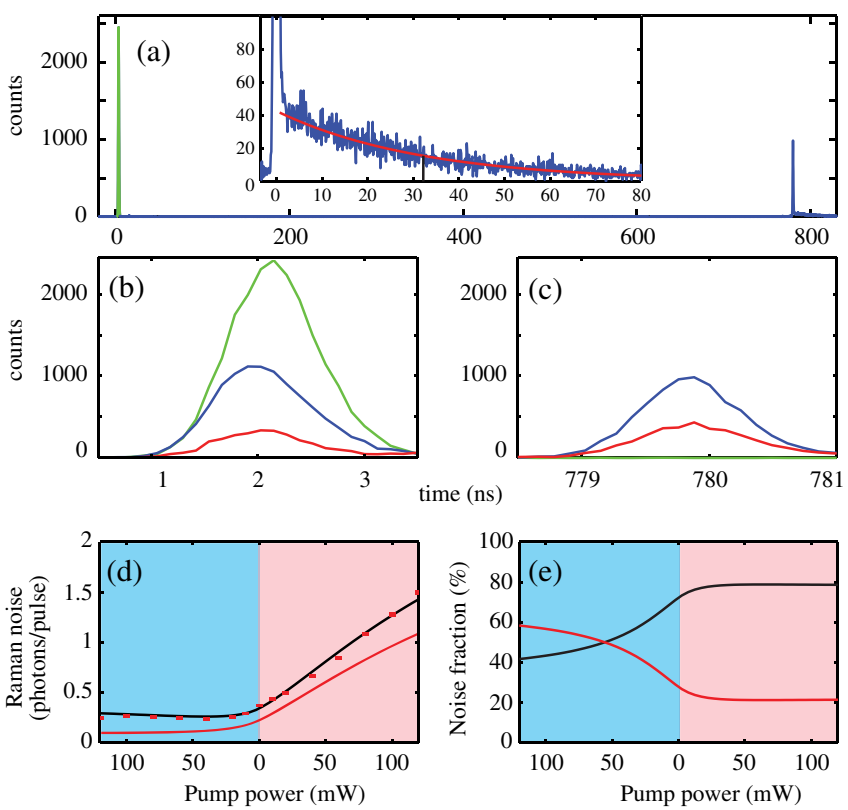

FIG. 3 (color online). Single-photon-level data. (a) Incident $\left(t_{1} \sim 0 \mathrm{~ns}\right)$ and retrieved $\left(t_{2} \sim 780 \mathrm{~ns}\right)$ pulse. The inset is a zoom of the retrieved pulse showing a fluorescence noise tail. (b),(c) Zooms around $t_{1}$ and $t_{2}$, showing the: incident signal (transmitted-retrieved signal with no control field) (green [light gray]); transmitted-retrieved signal with the control field (blue [dark gray]); and noise (control field only) (red [medium gray]). Histograms are accumulated over 360000 runs. (d) Blue (dark gray) shaded area: Optical pumping on the $|3\rangle \leftrightarrow|2\rangle$ (blue) transition. Red (medium gray) shaded area: Optical pumping on the $|1\rangle \leftrightarrow|2\rangle$ (red) transition. The red points are data and the black line is a theoretical prediction of noise due to spontaneous Raman scattering. The red solid line shows the predicted noise due only to Stokes scattering (e) Predicted fractional noise contributions from Stokes (black curve) and anti-Stokes (red curve) light. Theoretical details can be found in [26].

followed by an exponential tail, which is fluorescence noise from the excited state, $|2\rangle$ : this process is excited by the control field, even far from resonance, in the presence of atomic collisions. The red (medium gray) curve is a least-squares fit to the tail of the experimental data and yields an estimated lifetime of $32 \pm 2 \mathrm{~ns}$ (expected value $30.5 \mathrm{~ns}$ [30]; error bars derived from multiple experiments). While such collision-induced fluorescence limits the usefulness of other more narrowband roomtemperature memories (as shown in [11]), in our extremely broadband memory, the $30 \mathrm{~ns}$ time scale of these emissions is much longer than the duration of the readout event (set by the 300 ps pulse width and detector timing jitter). By time gating the detection, fluorescence therefore made a negligible contribution to the measured, instantaneous noise floor [Figs. 3(b) and 3(c)].

To understand where the instantaneous noise comes from, we then investigated its dependence on optical pumping. The blue (dark gray) shading in Fig. 3(d) indicates optical pumping on the $|3\rangle \leftrightarrow|2\rangle$ ("blue") transition, whereas the red (medium gray) shading represents 
optical pumping on the $|1\rangle \leftrightarrow|2\rangle$ ("red") transition. Increasing the pump power on the blue transition partially suppresses the noise, although it rapidly levels off at an average of 0.25 photons per pulse, while the noise level rises linearly with increasing pump power on the red transition.

These observations are well described by a simple noise model [26] based on spontaneous Raman scattering [31,32], in which Stokes and anti-Stokes photons scattered during the control pulse are both transmitted through our etalonbased filters and detected as noise. There is no applied magnetic field and Zeeman sublevels are not resolved due to Doppler broadening and the large control field bandwidth; we therefore neglect the magnetic structure of the hyperfine manifolds [26]. Since the model does not include collision-induced fluorescence [11] or leakage of the control, this suggests that these contributions are negligible. Furthermore, as shown in Figs. 3(d) and 3(e), around 60\% of the noise affecting the quantum memory is emitted at the anti-Stokes frequency (when operated with maximal blue pumping) and could be removed using further spectral filtering. This would already bring the true unconditional noise floor down to 0.1 photons per pulse resulting in an unconditional signal to noise ratio of 10:1 for single-photon retrieval. The remaining signal-frequency (Stokes) noise remains even if the optical pumping and spectral filtering are perfect and has its origin in four-wave mixing seeded by spontaneous anti-Stokes scattering. However, even this noise can be eliminated if the anti-Stokes channel can be suppressed or rendered much weaker than the Stokes channel, for instance by operating the memory closer to resonance, so that the anti-Stokes detuning is relatively much larger. Finally, although our fiber-coupled detection system is optimized for the mode of the signal, the noise is scattered mostly into the control mode [33,34], which suggests that the noise floor can be further reduced by angle tuning the control field [35]. These results show that the Raman memory, therefore, represents a genuine quantum-ready optical memory, functioning at room temperature.

In conclusion, we have demonstrated a broadband single-photon-level optical memory at room temperature, and conducted a detailed investigation of the unconditional noise floor. We have also extended the memory storage time to achieve a large time-bandwidth product, sufficient to dramatically improve heralded multiphoton rates from parametric sources - a crucial application for scalable linear optics quantum computing. This work shows that the far off-resonant Raman memory scheme makes it possible to implement a quantum-ready memory in warm atomic vapor, despite the results of previous studies of EIT that suggested otherwise $[11,12]$. Together with technological advances in microfabrication of vapor cells and hollow-core waveguide structures embedded on chips, Raman storage opens a path to an integrated, scalable quantum information technology architecture.
This work was supported by EPSRC through the projects QIP IRC (GR/S82716/01) and EP/C51933/01 and the European Community's Seventh Framework Programme FP7/2007-2013 under Grant No. 248095 for project QESSENCE, and the Royal Society. K. F. R. was supported by the Marie-Curie-Network EMALI and P. M. was supported by FASTQUAST. The authors would also like to acknowledge one of the referees for suggesting the qubit storage criterion for benchmarking the memory noise floor.

*k.reim1@physics.ox.ac.uk

[1] N. Gisin et al., Rev. Mod. Phys. 74, 145 (2002).

[2] L.-M. Duan et al., Nature (London) 414, 413 (2001).

[3] H. Briegel et al., Phys. Rev. Lett. 81, 5932 (1998).

[4] B. Julsgaard et al., Nature (London) 432, 482 (2004).

[5] T. Baluktsian et al., Opt. Lett. 35, 1950 (2010).

[6] B. Wu et al., Nat. Photon. 4, 776 (2010).

[7] K. Jensen et al., Nature Phys. 7, 13 (2010).

[8] I. Novikova et al., Phys. Rev. Lett. 98, 243602 (2007).

[9] M. D. Eisaman et al., Nature (London) 438, 837 (2005).

[10] J. Appel et al., Phys. Rev. Lett. 100, 093602 (2008).

[11] S. Manz et al., Phys. Rev. A 75, 040101 (2007).

[12] M. T. L. Hsu et al., Phys. Rev. Lett. 97, 183601 (2006).

[13] K. F. Reim et al., Nat. Photon. 4, 218 (2010).

[14] K. S. Choi et al., Nature (London) 452, 67 (2008).

[15] J. Simon et al., Phys. Rev. Lett. 98, 183601 (2007).

[16] D. N. Matsukevich et al., Phys. Rev. Lett. 97, 013601 (2006).

[17] C. Chou et al., Science 316, 1316 (2007).

[18] Y. Chen et al., Nature Phys. 4, 103 (2008).

[19] S. Chen et al., Phys. Rev. Lett. 97, 173004 (2006).

[20] M. P. Hedges et al., Nature (London) 465, 1052 (2010).

[21] H. de Riedmatten et al., Nature (London) 456, 773 (2008).

[22] J. Nunn et al., Phys. Rev. A 75, 011401 (2007).

[23] O. Cohen et al., Phys. Rev. Lett. 102, 123603 (2009).

[24] E. L. Raab et al., Phys. Rev. Lett. 59, 2631 (1987).

[25] M. Hosseini et al., Nature (London) 461, 241 (2009).

[26] See supplemental material at http://link.aps.org/ supplemental/10.1103/PhysRevLett.107.053603 for experimental details, arguments establishing the memory as quantum-capable, and calculations of magnetic dephasing and Raman noise.

[27] R. M. Camacho, P. K. Vudyasetu, and J. C. Howell, Nat. Photon. 3, 103 (2009).

[28] A. K. Ekert, Phys. Rev. Lett. 67, 661 (1991).

[29] S. Massar and S. Popescu, Phys. Rev. Lett. 74, 1259 (1995).

[30] D. A. Steck, http://steck.us/alkalidata (2009).

[31] M. Lukin et al., Phys. Rev. Lett. 82, 1847 (1999).

[32] N. Phillips, A. Gorshkov, and I. Novikova, J. Mod. Opt. 56, 1916 (2009).

[33] M. Raymer et al., Phys. Rev. A 32, 332 (1985).

[34] M.W. Sørensen and A.S. Sørensen, Phys. Rev. A 80, 33804 (2009).

[35] K. Surmacz et al., Phys. Rev. A 78, 033806 (2008). 